\title{
Prenatal diagnosis of $4 p$ deletion syndrome: A case series report
}

Dong Wook Kwak and Hyun Kyong Ahn*

Department of Obstetrics and Gynecology, Cheil General Hospital and Women's Healthcare Center, Dankook University College of Medicine, Seoul, Korea

The $4 p$ deletion syndrome, also known as Wolf-Hirschhorn syndrome, is a well-known genetic disorder caused by a partial deletion of the short arm of chromosome 4 . The great variability in the extent of the $4 p$ deletion and the possible contribution of additional genetic rearrangements leads to a wide spectrum of clinical manifestations. Herein, we present our experience with eight cases of $4 p$ deletion syndrome, ascertained prenatally between 1998 and 2016 at our hospital.

Key words: Wolf-Hirschhorn syndrome, 4p deletion, Prenatal diagnosis.

\section{Introduction}

The $4 p$ deletion syndrome, also known as Wolf-Hirschhorn syndrome (WHS; Online Mendelian Inheritance in Man [OMIM], 194190), is a well-known disorder caused by a partial deletion of the short arm of chromosome 4 [1]. The syndrome is named after K. Hirschhorn and U. Wolf, physicians who independently recognized the $4 p$-chromosome abnormality in the 1960s [2,3].

Clinical manifestations of $4 p$ deletion syndrome are variable. The most common findings include typical facial features (high forehead, prominent glabella, highly arched eyebrows, hypertelorism, short philtrum, distinct mouth, micrognathia), intrauterine/postnatal growth restriction, intellectual disability, hypotonia, seizures, skeletal anomalies (from medically significant malformation to minor anomalies), abnormal tooth development, and antibody deficiency. The less common findings include hearing loss, cleft lip/palate, structural brain anomalies, heart defects, and genitourinary tract malformations [4-6]. In addition, several cases of congenital diaphragmatic hernia, as well as those of lung and liver anomalies associated with $4 p$ deletion have been reported [6-8].

Most cases of WHS are caused by a pure deletion of $4 p 16$ without any other cytogenetic abnormality, but may also be caused by more complex cytogenetic findings such as $4 p$ mosaicism, chromosome 4 ring, or a derivative chromosome 4 resulting from either de novo or inherited unbalanced translocation $[9,10]$. The complexity of WHS-related cytogenetic findings is an important factor to account for phenotypic diversity.

Herein, we present eight cases of $4 p$ deletion syndrome, suspected upon observing abnormal signs on prenatal ultrasonography or aneuploidy screening tests and diagnosed with conventional cytogenetic testing.

\section{Case}

\section{Case 1}

A 34-year-old nullipara (G1 P0) was referred from a local clinic at 12 weeks of gestation due to fetal cystic hygroma. The

Received: 4 June 2017, Revised: 19 June 2017, Accepted: 20 June 2017, Published: 30 June 2017

*Corresponding author: Hyun Kyong Ahn, M.D.

Department of Obstetrics and Gynecology, Cheil General Hospital and Women's Healthcare Center, Dankook University College of Medicine, 23 Toegyero 46-gil, Jung-gu, Seoul 04619, Korea.

Tel: +82-2-2000-7127, Fax: +82-2-2278-4574, E-mail: hyunkyong.ahn@gmail.com

Conflict of interest: The authors declare that they do not have any conflicts of interest.

(c) This is an open-access article distributed under the terms of the Creative Commons Attribution Non-Commercial License (http://creativecommons.org/licenses/by-nc/4.0/) which permits unrestricted non-commercial use, distribution, and reproduction in any medium, provided the original work is properly cited.

c) Copyright 2017 by the Korean Society of Medical Genetics and Genomics 
family history was unremarkable. Sonographic examination at our hospital showed that the fetus not only had cystic hygroma, but also suspicious findings of cardiomegaly with abnormal left cardiac axis deviation and midline facial cleft. The patient was informed about the findings, and chorionic villus sampling was performed. The result of conventional chromosomal analysis was 46,XX,der(4)t(2;4)(p22;q15.3). A parental cytogenetic analysis was recommended, and the results of their karyotype were normal. When the mother came for post-test counseling, a fetal heartbeat was not detected on ultrasound. Termination of pregnancy was performed, but an autopsy was declined by the parents.

\section{Case 2}

A 31-year-old nullipara (G1 P0) was referred at 13 weeks of gestation for a positive double marker test for aneuploidy. The family history was unremarkable. After counseling, amniocentesis was performed. The result of trypsin-Giemsa banding revealed a karyotype with 46,XX,der(4)t(1;4)(q42.3;p15.2). Chromosomal analysis of the mother revealed a balanced translocation of $46, X X_{1} t(1 ; 4)(q 42.3 ; p 15.2)$, and the result of the father was normal. A second-trimester ultrasound was performed at 20 weeks of gestation, and the fetus was below the 3rd percentile. In addition, it showed cystic hygroma in the posterior neck space, cleft lip with palate, bilateral clubfoot, and findings suggestive of Dandy-Walker malformation (enlargement of the 4th ventricle and hypoplasia of the cerebellar vermis). After counseling, the parents decided to terminate the pregnancy. A female fetus weighing $311 \mathrm{~g}$ was delivered vaginally after medical induction. Fetal autopsy showed a left wrist flexion deformity and bilateral multicystic renal dysplasia, in addition to left cleft lip and palate, cystic hygroma, bilateral clubfoot, and DandyWalker malformation.

\section{Case 3}

A 36-year-old nullipara (G1 P0) was referred at 20 weeks of gestation due to abnormal ultrasound findings. The family history was unremarkable. A level II ultrasound showed that the fetus was in the 5th percentile, and he had a right paramedian cleft lip and palate, unilateral clubfoot, and cavum velum interpositum. The patient was informed, and amniocentesis was performed. Karyotype analysis revealed a deletion of the short arm of chromosome 4, 46,XY,del(4)(p15.3). A parental cytogenetic analysis was normal. After counseling, the patient was lost to follow-up.

\section{Case 4}

A 38-year-old primigravida (G3 P1) was referred at 20 weeks of gestation with a fetus suspected of having congenital diaphragmatic hernia. The family history was unremarkable. On the anatomic survey ultrasound, the estimated fetal weight was in the 11 th percentile and a thickened nuchal fold $(7.1 \mathrm{~mm})$, as well as a left-sided diaphragmatic hernia were detected. Karyotype analysis showed a deletion of the short arm of chromosome 4, 46,XY, del(4)(p15.3). Chromosomal analyses of the parents revealed normal karyotypes. After counseling, the patient was lost to follow-up.

\section{Case 5}

A 25-year-old nullipara (G2 P0) was referred at 18 weeks of gestation for fetal cystic hygroma on ultrasound. The family history was unremarkable, and the screening test for aneuploidy was negative. Evaluation at our hospital revealed the size of the fetus as below the 3rd percentile. Furthermore, she not only had a huge cystic hygroma of the neck and upper thorax, but also an aberrant right subclavian artery. An amniocentesis was performed and the result of trypsin-Giemsa banding revealed a karyotype with 46,XX,del(4)(p15.2). After genetic counseling, the patient was lost to follow-up.

\section{Case 6}

A 25-year-old nullipara (G3 P0) was referred at 18 weeks of gestation following a positive triple test for aneuploidy screening. There was no remarkable family history. We recommended karyotyping and an anatomic ultrasound scan, but she refused and was lost to follow-up. She showed up again at 27 weeks of gestation, and asked for karyotyping. Cordocentesis was performed, and fetal karyotype analysis led to the diagnosis of a $4 p$ deletion. The fetal chromosomal formula $46, X X, \operatorname{der}(4) t(4 ; 10)$ (p14;p12.3) was derived from a maternal translocated chromosome, 46, XX,t $(4 ; 10)(p 14 ; p 13.2)$. After patient counseling, she was lost to follow-up.

\section{Case 7}

A 28-year-old primipara (G2 P1) had been undergoing routine prenatal care since 6 weeks of gestation. The family history was unremarkable and the screening test for aneuploidy was normal. On performing the ultrasound at 21 weeks of gestation, the fetus was below the 3rd percentile, and she had a finding suspicious for an ankle deformity of the left foot. A follow-up ultrasound at 26 weeks of gestation showed hypoplasia of the inferior vermis, bilateral renal hypoplasia, as well as mild angula- 
tion deformities of both ankles. We suggested fetal karyotyping based on the ultrasound findings, and cordocentesis was performed at 28 weeks of gestation. The result of trypsin-Giemsa banding revealed a karyotype with $46, X X, d e l(4)(p 15.2)$. On the day following cordocentesis, she could not feel any movement of the fetus. Intrauterine fetal demise was diagnosed, and termination of the pregnancy was performed. A dead female fetus weighing $860 \mathrm{~g}$ was delivered vaginally after medical induction, and an autopsy showed cleft palate, hypoplasia of both kidneys, incomplete lobation of the right lung, and a single lobe of the left lung. Chromosomal analyses of the parents were normal.

\section{Case 8}

A 33-year-old primipara (G5 P1) had been undergoing routine prenatal care since 6 weeks of gestation. The family history was unremarkable, and the screening test for chromosomal aneuploidies was normal. During the second-trimester ultrasound, structural abnormalities were not detected, but the fetus was below the 3rd percentile. A follow-up ultrasound was performed at 29 weeks of gestation, and fetal intrauterine growth restriction (IUGR) had worsened (the estimated fetal weight was 902 g) without structural abnormality. Karyotyping was suggested due to IUGR, and amniocentesis was performed. The result of trypsin-Giemsa banding revealed a karyotype with 46,XX,del(4) (p15.3). A parental cytogenetic analysis showed normal karyotypes. After genetic counseling, the patient was lost to followup.

\section{Discussion}

We report eight cases of $4 p$ deletion syndrome that were prenatally diagnosed. WHS is a rare but well-described congenital condition consisting of multiple anomalies, caused by partial deletion of the short arm of chromosome 4. In 1961, just 2 years after the chromosomal basis of Down syndrome was described, Hirschhorn et al. [2] presented a case report in Mammalian Chromosomes Newsletter of a child with midline defects and an obvious deletion of the short arm of one of the B-group chromosomes. The estimated incidence of WHS is around 1/50,000 [4]. However, this is likely underestimated because of underrecognition or misdiagnosis of affected individuals [4]. In this study, there were four females and four males, but WHS is more common in females than in males, with a male-to-female ratio of $1: 2$ [11].

In the literature, about $55 \%$ of $4 p$ deletion syndrome cases result from an isolated $4 p$ deletion; about $40-45 \%$ of affected individuals have an unbalanced translocation (de novo or inherited from a familial balanced rearrangement), characterized by both a deletion of $4 p$ and a partial trisomy of a different chromosome arm. In the remaining cases, there is a complex rearrangement leading to a $4 p 16.3$ deletion (e.g., chromosome 4 ring) [5]. Unbalanced translocations involving the short arms of chromosomes 4 and 8 appear with high frequency in several large case series of WHS patients. In our study, six cases (75\%) were de novo documented deletions and two cases (25\%) involved a $4 p$ deletion resulting in a derivative chromosome 4 , derived from maternal translocation.

$4 p$ deletion syndrome is a multiple malformation syndrome, characterized by mental and developmental defects resulting from the absence of a segment of the chromosome 4 short arm (4p16) [12]. Clinical severity differs according to the size of the deletion among affected individuals. Studies suggest that larger deletions tend to cause more severe intellectual disability and physical abnormalities than smaller deletions [13].

The majority of prenatally diagnosed cases have incidentally been detected after an indicated amniocentesis for advanced maternal age [14], a positive aneuploidy screening test [15], parental unbalanced translocation $[16,17]$, or IUGR with or without associated fetal malformations $[14,18]$.

In this study, karyotyping was performed due to positive aneuploidy screening tests in two cases and abnormal findings on prenatal ultrasonography in the other cases. Furthermore, between the two cases with abnormal aneuploidy screening, one case revealed multiple structural anomalies on second-trimester ultrasound, and the other patient did not receive an ultrasound at our hospital due to her refusal. Therefore, fetuses with $4 p$ deletion syndrome might have an increased likelihood of abnormal ultrasound findings.

Among seven fetuses with abnormal ultrasound findings, three had a midline facial cleft. Cystic hygroma was also detected in three cases: two cases were diagnosed during the second trimester and one case during the first trimester. Additionally, many abnormalities were identified by ultrasound or autopsy (Table 1), including the following: hypoplasia of the inferior vermis with a prominent 4th ventricle (Dandy-Walker malformation), hypoplasia of both kidneys, angulation deformity of the ankle, clubfoot, diaphragmatic hernia, nuchal fold thickening, and aberrant right subclavian artery. In our study, all six cases for which a second-trimester ultrasound was performed showed findings suspicious for IUGR, and the fetal size in four of them was below the 3 rd percentile. Notably, IUGR was the only finding in case 8. 


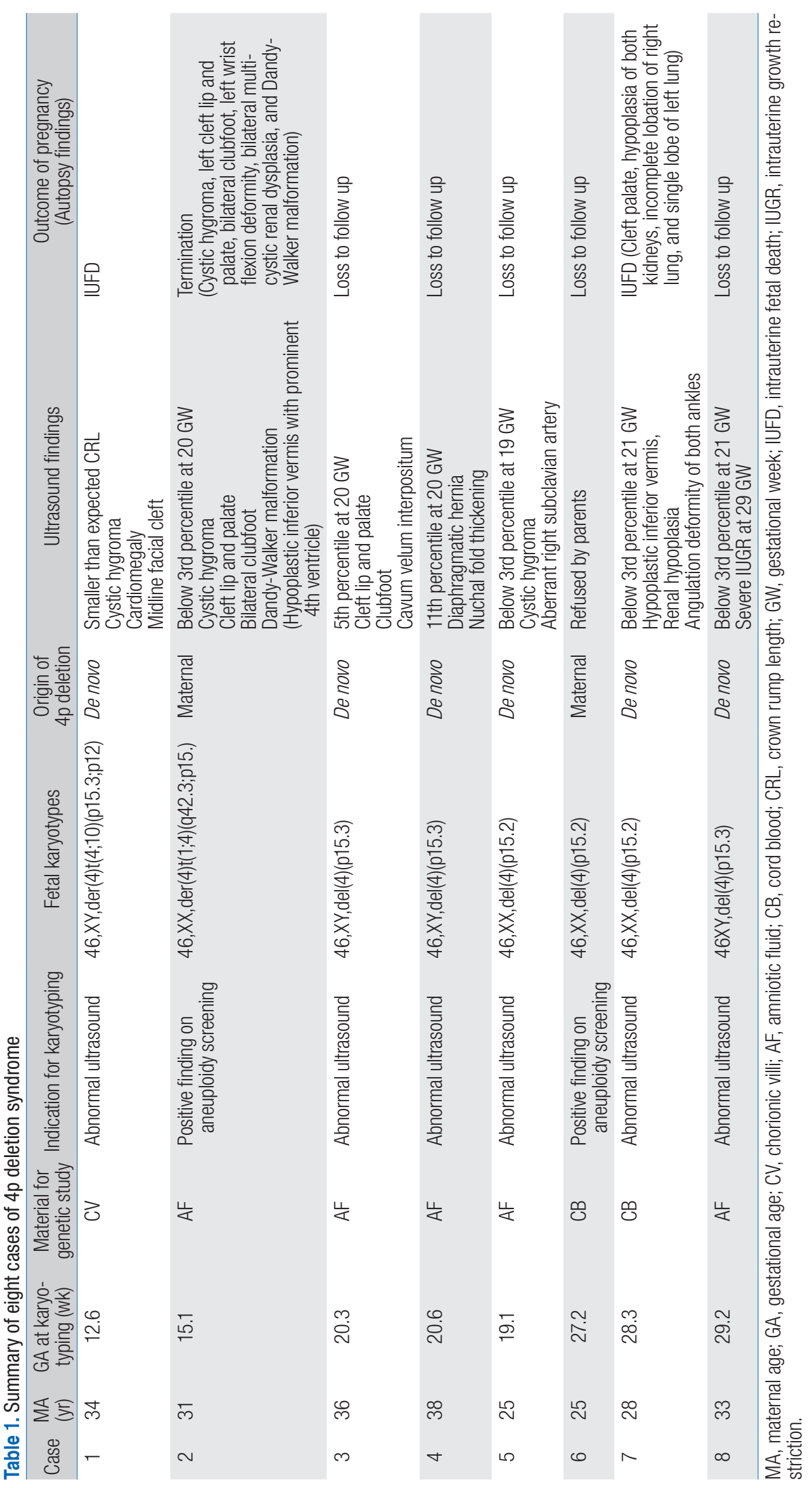


In conclusion, our prenatal findings of the $4 p$ deletion syndrome are variable. However, in most cases of WHS, a severe growth restriction was observed from the second trimester, and it was observed to be present either as an isolated finding or in association with facial cleft, cystic hygroma, renal or skeletal abnormalities. Additionally, we observed isolated finding of congenital diaphragmatic hernia may also be indicative of the $4 p$ deletion syndrome, and the Dandy-Walker malformation could be present in WHS in this study. We hope that presenting our experiences will be helpful to obstetricians who counsel patients with these findings.

\section{Acknowledgements}

We would like to thank the Laboratory of Medical Genetics, Cheil General Hospital and Women's Healthcare Center.

\section{References}

1. On-Line Mendelian Inheritance in Man, OMIM. [http://www.ncbi.nIm. nih.gov/omim?term=WHS]

2. Hirschhorn K, Cooper HL, Firschein IL. Deletion of short arms of chromosome 4-5 in a child with defects of midline fusion. Humangenetik 1965;1:479-82.

3. Wolf $U$, Reinwein $H_{1}$ Porsch $R$, Schröter $R$, Baitsch $H$. [Deficiency on the short arms of a chromosome No. 4]. Humangenetik 1965;1:397413. German.

4. Battaglia A, Carey JC, Wright TJ. Wolf-Hirschhorn (4p-) syndrome. Adv Pediatr 2001;48:75-113.

5. Battaglia A, Carey JC, South ST. Wolf-Hirschhorn syndrome: a review and update. Am J Med Genet C Semin Med Genet 2015;169:216-23.

6. Tachdjian G, Fondacci C, Tapia S, Huten Y, Blot P, Nessmann C. The Wolf-Hirschhorn syndrome in fetuses. Clin Genet 1992;42:281-7.

7. Basgul A, Kavak ZN, Akman I, Basgul A, Gokaslan H, Elcioglu N. Prenatal diagnosis of Wolf-Hirschhorn syndrome (4p-) in association with congenital diaphragmatic hernia, cystic hygroma and IUGR. Clin
Exp Obstet Gynecol 2006;33:105-6.

8. Prunotto G, Cianci P, Cereda A, Scatigno A, Fossati C, Maitz S, et al. Two cases of hepatic adenomas in patients with WolfHirschhorn syndrome: a new rare complication? Am J Med Genet A 2013;161A:1759-62.

9. Zollino $M$, Lecce $R$, Fischetto $R$, Murdolo $M$, Faravelli $F$, Selicorni $A$, et al. Mapping the Wolf-Hirschhorn syndrome phenotype outside the currently accepted WHS critical region and defining a new critical region, WHSCR-2. Am J Hum Genet 2003;72:590-7.

10. Dallapiccola B, Mandich $P$, Bellone E, Selicorni A, Mokin V, Ajmar $F_{1}$ et al. Parental origin of chromosome $4 p$ deletion in Wolf-Hirschhorn syndrome. Am J Med Genet 1993;47:921-4.

11. Coles K, Mackenzie M, Crolla J, Harvey J, Starr J, Howard F, et al. A complex rearrangement associated with sex reversal and the WolfHirschhorn syndrome: a cytogenetic and molecular study. J Med Genet 1992;29:400-6

12. Wilson MG, Towner JW, Coffin GS, Ebbin AJ, Siris E, Brager P. Genetic and clinical studies in 13 patients with the Wolf-Hirschhorn syndrome [del(4p)]. Hum Genet 1981;59:297-307.

13. Pokale YS, Jadhav AM, Kate U. Wolf-Hirschhorn syndrome: a case demonstrated by a cytogenetic study. Indian J Hum Genet 2012;18:117-8

14. Blunt $S$, Berry AC, Seller MJ, Williams CA. Prenatal recognition of $4 p$ syndrome. J Med Genet 1977;14:232-3.

15. Lee MH, Park SY, Ryu HM, Hong SR, Lee YH, Choi SK. Prenatal diagnosis of the Wolf-Hirschhorn syndrome. J Genet Med 1998;2:49-51.

16. Vamos E, Pratola D, Van Regemorter N, Freund M, Flament-Durand J, Rodesch F. Prenatal diagnosis and fetal pathology of partial trisomy 20P-monosomy 4P resulting from paternal translocation. Prenat Diagn 1985;5:209-14.

17. Kohlschmidt N, Zielinski J, Brude E, Schäfer D, Olert J, Hallermann C, et al. Prenatal diagnosis of a fetus with a cryptic translocation $4 p ; 18 p$ and Wolf-Hirschhorn syndrome (WHS). Prenat Diagn 2000;20:152-5.

18. Snijders RJ, Sherrod C, Gosden CM, Nicolaides KH. Fetal growth retardation: associated malformations and chromosomal abnormalities. Am J Obstet Gynecol 1993;168:547-55. 